\title{
Conflicts between Authenticity and User Retrieval in Classification Design
}

\author{
Julia Bullard \\ The University of Texas at Austin \\ School of Information
}

The field of knowledge organization theory shows major divides in priorities and conclusions: whether the core of classification is structure or meaning (Beghtol, 1986; Mills, 2004; Spärck Jones, 1970), whether a classification designer can or should rely solely on the literature to determine terminology (Fidel, 1994; Hulme, 1911; Mai, 2005), and whether it is the global principles (Ranganathan, 1962) or the individual classification designer's knowledge and insight (Albrechtsen \& Pejtersen, 2003; Feinberg, 2007; Hjørland, 2002) that creates a good system. The field of classification research offers no clear consensus on how classification construction occurs in practice or its ideal version (Park, 2008). Nor do we understand how classificationists negotiate conflicts between these principles and approaches in daily work. For example, if literary warrant is insufficient to make semantic decisions (Fidel, 1994; Mai, 2005), how do classification designers justify and reconcile - to themselves, their institutions, and their users - the inevitable use of personal and contextual information with the belief that the system should represent the documents without bias? Similarly, at what point (if any) in a domain analysis process does the classification designer encounter the impact of his or her system on the field being organized, and how does he or she seek to minimize or justify this distortion?

Our literature presents a host of different approaches and assumptions regarding the potential and purpose of classification systems; we can extend this research by finding out how these are embodied in the lived experience of classification designers. In this paper, I use the term "classification designer" to refer to specialists who create and maintain controlled vocabularies for knowledge organization systems, regardless of the specific genre of schema (classification, thesaurus, or otherwise), and do not specifically address classifiers or indexers who apply these schemes to objects. I adopt observational and ethnographic methods, previously demonstrated by Park (2008) and Mayernik (2011), to pursue research questions key to our field, such as: How is design is accomplished in an environment of multiple goals and obligations? and, How do classification designers understand their impact on organizing systems that shape users' experiences of documents and concepts? In contrast to accounts of classification design that recount past design decisions and are subject to retrospective rationalization, observing ongoing design work is likely to reveal conflicts and compromises that speak to ongoing and fundamental divides in our field.

I provide an example of how observational methods can reveal such conflicts in classification design by focusing on the daily design decisions made in extending and maintaining a live classification system. Using preliminary data from my dissertation, I focus on a single set of decisions in a classification design project. I present a textual analysis of online discussions between classification designers, accessed and collected as part of ongoing participant observation in the project. This snapshot of the 
Julia Bullard. 2015. Conflicts between Authenticity and User Retrieval in Classification Work.

In Smiraglia, Richard P., ed. "Proceedings from North American Symposium on Knowledge Organization", Vol. 5.

Los Angeles, CA, pp. 16-24.

designers' deliberation demonstrates persistent divisions between classification-for-retrieval and classification-as-representation.

\section{Classification Design Site}

The Fanwork Repository is an online fanfiction library in which a group of over 200 volunteers manage a classification system for the organization and retrieval of user-submitted works. The collaborative classification project in this system is the design and maintenance of a curated folksonomy - a system of synonyms and relationships that addresses some of the major shortcomings of an unregulated folksonomy. This knowledge organization schema most closely resembles a thesaurus, with the terminology and concepts originating from authors' and users' indexing or tagging actions. Each month, writers add thousands of works to the site, each with tags attached. Freeform tags submitted by content creators for their works are then made synonyms, broader terms, and narrower terms to existing tags and to tags created by the classification designers. Classification designers in this system therefore control the collocation of documents with shared concepts regardless of variant word choice and influence future word choice by populating autocomplete suggestions lists. As a participant observer, I have contributed to this system as a volunteer for two years and have access to system data, mailing list conversations, and persistent chat room logs for analysis.

The nature of the collected works in this site stands out as a sharp departure from the common domains of professional classification work: the works are fictional, user-generated, and exist outside traditional commercial, educational, or governmental institutions. However, the classification work around these works embodies many of the primary goals and challenges of classification systems. First, the system must be usable and even intuitable by authors who add works as well as users who seek works. Second, The Fanwork Repository seeks as its audience the universe of potential fanfiction authors and readers. As a "universal" classification system, work on The Fanwork Repository contains many of the challenges of mainstream systems like the Library of Congress Classification, which must remain relevant across many fields and over decades of work. Like these familiar universal systems, The Fanwork Repository covers such a broad range of concepts, documents, and potential users that the classification designers cannot reasonably check each decision against the universe of possible effects and stakeholders, driving the development of general guidelines and principles. Third, the system is large and rapidly growing, necessitating a team of classification designers who must coordinate the growth and maintenance of the classification system. The work is collaborative and involves both the division of domains of responsibility among classification designers as well as the coordination of guidelines and precedent across all domains. This has largely methodological advantages, in that the classification designers routinely explain, argue, and justify their decisions among members the group, providing opportunities to observe and analyze these explanations in a naturalistic setting.

Typically, the work of classification design in this setting is a constant pace of sorting new tags according to umbrella domains (determined by the original media, such as "Harry Potter - J.K. Rowling") and creating links to existing tags where possible. Occasionally, promoted writing events in the larger fanfiction community present a different dynamic in which classification designers expect a surge of tags in a known domain, motivating classification designers to gather and discuss likely issues in preparation. In January of 2014, The Fanwork Repository hosted such a community event - a "ficathon" in which 
participants could nominate source works which other participants would choose from in writing stories for the event. The subject of this event was works that exist within fictional works, such as the television series Pleasantville that provides the setting for the movie Pleasantville or The Itchy and Scratchy Show, a television series that the children on The Simpsons watch religiously. In hosting the event, The Fanwork Repository not only provided a location for the stories themselves but also facilitated the creation of a set of tags event moderators used to coordinate the event. The volunteer classification designers discussed this event in detail over the mailing list. The deliberation around this event was about finding a solution that would both fit currently known tags and could be reasonably extrapolated to cover expected or likely future tags. In the following textual analysis of the designers' discussion, I focus on how classification designers pose, argue for, and argue against different solutions in the form of terms and term relationships.

The classification designers discussed this event in detail. Part of the purpose of event tag sets is to give the classification designers an opportunity to manage these tags before the majority of participants add their works to the site. Since events involve many related works being posted (and read) in a relatively short span of time, it is particularly urgent that classification designers integrate these new tags into the curated folksonomy. Managing the tag sets for these events is a different type of work and challenge for the classification designers than the typical work of the site. Maintenance and reaction are the dominant modes of work in the site: classification designers cannot designate tags as preferred terms or place them in relationships with other terms until a user creates them as a tag. Some types of tags - such as generic tags not belonging to any one fandom - have additional rules requiring classification designers to wait until there are 3 or more uses of a given concept before creating a preferred term. Managing event tag sets, on the other hand, is instead a matter of planning and creating: in the early stages of an event, classification designers have the chance to make decisions about tags and their relationships before they are "live" in the system. This is especially true in cases like that of the Fiction-within-Fiction event, which, given its subject matter, targets fandoms and concepts that have not yet been used in the site. For classification designers, this is an uncommon opportunity to develop guidelines and apply them consistently from the outset of a trend. For the few Fiction-withinFiction fandoms that existed in the site before this event, classification designers made ad hoc interpretations of the existing guidelines to handle these atypical cases; during the early stages of this event, classification designers discussed possible guidelines based on the wide array of nominated fandoms. As with the typical maintenance-and-reaction work, the deliberation around this event was both about finding a solution that fits the currently known tags and could be reasonably extrapolated out to cover expected or likely future tags.

\section{Observational Data Sources}

My primary data sources for the classification work around this fandom event are the mailing list threads discussing the event (a single discussion separated into two separate threads because of a subject line addition of "Re:" part way through), the chat room discussions from the same time period discussing the event, the publicly available system data denoting the final designations of all the tags in the event's tag set, and the internal discussions between classification designers attached to these tags. In this section I describe each type of observational data and how it informs analysis. Except for the 
system data, which indicates the current or final disposition of the terms and their relationships, none of this data would be available to me except through my agreement to participate in the community as a classification designer. In presenting the results of my analysis of a small window of activity in this setting, I will reveal conflicts and compromises not apparent from the finished system alone and that might be minimized in retrospective accounts by the designers themselves.

The mailing list discussion was started by a classification designer responsible for managing the event's tag set. This classification designer (referred to as Toni here) asked for the group's opinion on four questions:

- In which media type [Cartoons, Books, Other Media, etc.] should the Fiction-withinFiction fandoms be categorized, considering that some differ in media type from their source fandoms?

- Should they be automatically subtagged to their source fandoms if they are made preferred terms?

- Should they, at any point, be made synonymous to their source fandoms?

- If they need disambiguation, do we do so as if they were a regular fandom, by adding their media type, by adding the title of their source fandom, or by adding "fictional", "imaginary", or another chosen term?

The discussion in the mailing list addressed each of these questions, though a few messages included or were only messages of excitement regarding the event itself. More than 30 classification designers participated across a total of 73 messages. A typical message was of the following format:

Jess [email address][Date \& Time]

I'm going to object to the idea of the disambigs including the word fictional if imaginary for two reasons. (1) why is something any more fictional or imaginary just because it has been sourced out of a work that was fiction itself? (2) I have seen the some of the nominations rolling in and they include that ever-present sticky-wicket on [The Fanwork Repistory] of religion and lore and identity fandoms. The Gospel Of Eve has been nominated. It is lost source text apocrypha from the bible. I think it would be a really bad idea to apply words like imaginary or fiction to a part of anyone's religious or cultural identity just because the source work itself is no longer extant. That's my two cents for the minute. Take it or leave it as you like as always

- Jess

I will return to Jess' message in discussing the themes of retrieval and authenticity, below.

Chat room discussions around the event were primarily excitement about the event and speculation regarding which fandoms would be included in the event. Even in the chat space exclusively 
Julia Bullard. 2015. Conflicts between Authenticity and User Retrieval in Classification Work.

In Smiraglia, Richard P., ed. "Proceedings from North American Symposium on Knowledge Organization", Vol. 5.

Los Angeles, CA, pp. 16-24.

available to the classification designers, discussion was from the point of view of a participant in the fandom community rather than a designer who would have input on the management of its associated tags. Classification designers in the mailing list discussion often referred to example cases of fandoms either predating the event, already added to the event, or reasonable targets of the event. Information about the classification decisions made about these specific tags can be found through the system. Publicly available system data shows the media designations for all of the fandoms within the event's tag set, so that at a glance it is possible to see that Satan's Alley, The Rural Juror, and Blasto are all categorized as movies, though their source fandoms are a movie, a television series, and a video game series, respectively. Navigating the tag system from any of these shows how it is currently related to other tags. Even though Satan's Alley has several stories attached, and its source fandom, Tropic Thunder, is also in the repository, it has no meta- or subtags. Wormhole X-Treme, however, is subtagged to the television series fandom Stargate SG-1, its source fandom.

Many of these fandom tags have comments attached, visible only to classification designers and not to the typical site users. Some comments predate the Fiction-within-Fiction event while others closely follow and refer to the discussion over the mailing list. For example, on the fandom tag for Wizardy Herbert, a subtag of the fandom tag Homestuck, there are two comments. The first, dated 6 months before the event, reads: "[Sender Name] [Date \& Time] I split this off from Homestuck because there's actually a partial draft of the Wizardy Herbert novel available. It's a story written by one of the characters in Homestuck, but is actually a separate canon." A second comment, written by the same classification designer and dated 3 days after the beginning of the mailing list discussion reads: "[Sender Name] [Date \& Time] Moving this out of "Cartoons \& Comics \& Graphic Novels" to "Books \& Literature" per the ongoing conversation on the ML [mailing list], and so as not to confuse people browsing [Fictionwithin-Fiction's] tagset". Some comments left in this way form threaded discussions. While the mailing list discussion is the central data source for the following analysis, the chat room transcripts and system data provide background and contextual data used by the classification designers at the time of the discussion. Similarly, my prolonged engagement in this volunteer workforce provides the necessary information to interpret the meaning of some shorthand forms of argument and references to established principles.

Together, these sources of data provide multiple perspectives on these classification design decisions. The chat logs and the live system show how these fandom events and their expression in the system appear to users. The documentation through internally-visible comments provide insight into the history of these issues and the institutional knowledge shared by the designers. The mailing list shows the issues as the designers understood them at the outset of this event. Along with expertise in knowledge organization principles, these sources provide the benefit of a participant's understanding of the discussion along with an observer's distance to witness rather than influence or rationalize.

\section{Authentic vs. Effective Classification}

Even in a system and collection as novel as that of The Fanwork Repository, the perennial poles of user retrieval and authenticity found in knowledge organization theory are matters of daily debate. For this paper, I wish to highlight one outcome of my analysis of this event, the importance of this primary conflict of knowledge organization principles to various design decisions. In the course of the 
discussion around this event, classification designers came to argue over two competing interpretations of the purpose of a classification system: to authentically represent the collection or to facilitate retrieval. Even the first interpretation can have multiple versions: to represent the organization of things as they really are in the world or according to the documents' or authors' own logic. This common conflict in this community reflects long-standing debates in knowledge organization theory and design. In the Fiction-within-Fiction event, this conflict complicated two decisions: what name to give to these types of fandoms and what relationship should these fandoms have to their source fandoms in the classification system.

The first conflict is related to the issue of disambiguation. Jess' message, above, pointed out two flaws in using "(Fictional)" as the clarifying term to denote the difference between these fandoms and those of conventional works. The second - that some nominated fandoms should not be described as fictional - was resolved as being out of the scope of the discussion. The first was reiterated by various classification designers, that "fictional" is a largely meaningless term in the context of fanfiction. Even if the classification designers accept that originating from within another work makes a fandom "more fictional", they cannot expect users to make the same distinction, especially as they encounter these fandom terms alone and not in the aggregated view of the classification designer. Though it was originally her suggestion, Toni later reveals that her hesitation in using "(Fictional)" is that users might treat it as a distinction from real-person fiction, stories based on personalities in the real world. Heidi, another classification designer, points out that both "(Fictional)" and "(Imaginary)" are poor choices for long-term guidelines since "there is precedent for "fictional" fandoms to become real in their own right. (Castle \& Jessica Fletcher's novels for example)".

Classification designers offered alternative suggestions for a disambiguating term to be used for all Fiction-within-Fiction fandoms, or those that lack an easy solution such as the author name attached to The Princess Bride. These suggestions included "in-universe", "parafandom", "metafandom", and "metafictional". Despite finding "metafictional" to be the most accurate, specific term to describe these types of fandoms, classification designers still objected to the use given the likelihood of confusing users. "Meta" already has two common uses within the site's community: metatags, such as "Marvel Cinematic Universe", under which are collected multiple related fandom terms, such as "Iron Man (Movies)", and meta, which is used to mean a discussion of fandom itself or stories in which characters talk about their worlds as fandom. Correctness, in the classification system, must be judged from the perspective of experts as well as typical users, who will use these tags without the context of the classification designers' discussion.

The second conflict between authenticity and retrieval speaks to the structure of classification systems. Though the curated folksonomy is not bound by strict classification rules, classification designers are concerned that the hierarchical structure of meta- and subtags follows basic rules of logic. A subtag to the metatag "Marvel Cinematic Universe" must be part of that universe, and the subtags of that subtag must also pass the test as belonging to the "Marvel Cinematic Universe". Classification designers often examine larger hierarchical structures within the classification system for instances of "concept drift", a telephone-game effect whereby successive connections between tags stretch the "isa" relationship between top- and bottom-level tags. The concern for the sanctity of the hierarchical 
Julia Bullard. 2015. Conflicts between Authenticity and User Retrieval in Classification Work.

In Smiraglia, Richard P., ed. "Proceedings from North American Symposium on Knowledge Organization", Vol. 5.

Los Angeles, CA, pp. 16-24.

relationship is not just conceptual, it is also functional. Searching or filtering by metatags, by default, returns works tagged with any of its subtags.

In the course of the discussion on Toni's question regarding subtagging Fiction-within-Fiction works to their source fandoms, some classification designers supported an alternative option, making both the source fandom and the Fiction-within-Fiction fandom subtags of a new, common metatag. Some metatags of this type already exist in the system. For example, Sherlock Holmes \& Related Fandoms is an umbrella fandom over the Arthur Conan Doyle books, the Guy Ritchie film series, and Elementary, a current television series, among other works. Classification designers who supported parallel or "sister" relationships rather than meta- and subtag relationships between the source and Fiction-within-Fiction fandom did so on the basis of precision in retrieval: by making the two fandoms parallel, it would be possible for users to selectively browse and search across both fandoms or exclusively view one fandom at a time. The connecting through the metatag would not only make searching across both easy, but would also signify to users the association between the two fandoms.

The classification designers who supported the original suggestion of subtagging the Fictionwithin-Fiction fandom to the source fandom also argued that it would support retrieval, though this solution would prevent users from viewing only those works from the source fandom; the Fictionwithin-Fiction fandom would always be folded into their search results. The advantage of this approach, however, was in the authenticity of the classification relationship to the conceptual relationship between the fandoms. Fiction-within-Fiction fandoms have a part-whole relationship to their source fandoms. They are not parallel to their source fandom - they are within it. For classification designers and users who consider the hierarchical relationships in the curated folksonomy as representative of relationships between the represented concepts, this is the appropriate solution. However, this solution does not allow the same relationship to play out in the user's experience of searching and browsing: though the Fiction-within-Fiction fandom is dependent on its source for its existence, as a subtag it can be separated out as its own category in search while users cannot view the source fandom alone. Precision in searching favors only the smaller, dependent fandom.

In the live version of the system, classification designers overwhelming made the choice based on authenticity for the fandoms within their domain of responsibility. Some, such as Satan's Alley or Celestial Emporium of Benevolent Knowledge, have no relationship to their source fandoms, which may be a product of the classification designers being unable to decide on either solution and therefore making none at all. Others, such as Wizardy Herbert, are made subtags to their source fandoms. I could find no cases of fandoms made parallel in the hierarchy, even when the previously established tag context would lend itself to this, as in the case of many books arranged under a single author.

\section{Implications for Knowledge Organization Theory}

The decisions and types of arguments presented through the Fiction-within-Fiction event reveal the tension between the ideal system the classification designers would like to design and the messy system the users create. While classification designers discuss these issues in detail, weighing the choice of terms and terms relationships based on logic, precedent, and even elegance, users are not privy to the same discussions. Classification designers, through their own domains of responsibility and in 
engaging with other designers on system-wide principles, experience the system as a whole. Users' experience with tags is more likely to be limited to the specific tags they add to stories or that they employ in searching and browsing. The discussions I have highlighted in this analysis show how classification designers encounter and remind themselves of the users' imperfect understanding of the system. In suggesting disambiguating terms to attach to Fiction-within-Fiction fandoms, the users' understanding is the primarily goal: any solution which misleads or confuses users does not serve the purpose of distinguishing between similar terms to help users apply them correctly.

Within classification theory, schools of thought differ on the centrality of user data and the user perspective on the design of a classification system. The movement towards user-centered or participatory design, for example, privileges the user's perspective and argues that, since the system is made for users, it must match their language and needs. Others, from Henry Bliss (1929) to Birger Hjørland (2013), argue that a system best serves users when that system represents the relationships between concepts correctly, not only in spite of but because of users' subsequent efforts to adapt their understanding to it. In the case of the live classification system used for The Fanwork Repository, the relationship is messier than either of these versions. User data informs the classification system and is through the folksonomy itself - the raw material for the classification system. Classification designers explicitly recognize that the system exists to support user retrieval. Users, however, are a large and diverse group, and the "behind-the-scenes" nature of the classification work means that designers cannot reach out to users directly but must rely on the system itself to instruct users on its meaning and nuances, leading the designers to compromise between the ideal or authentic relationships and those that are most likely to survive contact with users.

It remains difficult to bridge the perspectives of classification designers and users in KO scholarship. As designers ourselves, we contribute to the literature by recounting design experiences, demonstrating the logic behind particular choices, and championing our approaches and goals. As readers of others' systems, we contribute to the literature by looking closely at the system as a whole and exploring the - perhaps unintended - impact of particular choices. In this analysis, I used an observational approach to focus on daily design decisions and how they call upon knowledge organization theory. In environments - such as the site I describe here - where designers routinely deliberate and collaboratively develop guidelines, such analysis reveals a vivid picture of the connection between theory and practice.

\section{References}

Albrechtsen, H., \& Pejtersen, A. (2003). Cognitive work analysis and work centered design of classification schemes. Knowledge Organization, 30(3-4), 213-227. Retrieved from http://cat.inist.fr/?aModele=afficheN\&amp;cpsidt=16103913

Beghtol, C. (1986). Semantic validity: concepts of warrant in bibliographic classification systems. Library Resources \& Technical Services, 30(April/June), 109-125. Retrieved from http://downloads.alcts.ala.org/Irts/Irtsv30no2.pdf\#page=3 
Bliss, H. E. (1929). The organization of knowledge and the system of the sciences. New York, NY: Holt \& Co.

Feinberg, M. (2007). Hidden bias to responsible bias: an approach to information systems based on Haraway's situated knowledges. Information Research, 12(4). Retrieved from http://informationr.net/ir/12-4/colis07.html

Fidel, R. (1994). User-centered indexing. Journal of the American Society for Information Science, 45(8), 572-576. Retrieved from http://lis571-help.wdfiles.com/local--files/websites/user centered indexing.pdf

Hjørland, B. (2002). Domain analysis in information science: Eleven approaches - traditional as well as innovative. Journal of Documentation, 58(4), 422-462. doi:10.1108/00220410210431136

Hjørland, B. (2013). User based and cognitive approaches to knowledge organization. Knowledge Organization, 40(1), 11-27.

Hulme, E. W. (1911). Principles of book classification, Part 3. Library Association Record, 13, 444-449.

Mai, J.-E. (2005). Analysis in indexing: document and domain centered approaches. Information Processing \& Management, 41(3), 599-611. doi:10.1016/j.ipm.2003.12.004

Mayernik, M. S. (2011). Metadata realities for cyberinfrastructure. University of California Los Angeles.

Mills, J. (2004). Faceted classification and logical division in information retrieval. Library Trends, 52(3), 541-570. Retrieved from https://www.ideals.illinois.edu/bitstream/handle/2142/1035/L?sequence=2\#page=173

Park, O. N. (2008). Current practice in classification system design: An empirical investigation of classification system design team practice. University of Washington.

Ranganathan, S. R. (1962). Elements of Library Classification. New York: Asia Publishing House.

Spärck Jones, K. (1970). Some thoughts on classification for retrieval. Journal of Documentation, 26(2), 89-101. doi:10.1108/eb026488 